The $3^{\text {rd }}$ Conf. of SSFOP "Recent Techniques in Ornamental Plants Scope”, Cairo, Egypt, 26/2/2017

Scientific J. Flowers \& Ornamental Plants

www.ssfop.com/journal

ISSN: 2356-7864

\title{
GROWTH AND YIELD OF LOCAL CELERY LINES IN RESPONSE TO FOLIAR APPLICATION OF YEAST AND SALICYLIC ACID
}

\author{
${ }^{* *}$ Sakina I. Ismail*, Soher E. El-Gendy ${ }^{* *}$, Nada M. Darwesh \\ * Medicinal and Aromatic Plants Res. Dept., Hort. Res. Inst., ARC, Giza, Egypt. \\ ** Cucurbits and Cross-Pollinated Vegetable Crops Res. Dept., Hort. Res. Inst., ARC, Giza, Egypt.
}

\begin{abstract}
The present study was carried out in two successive seasons of 2013/2014 and 2014/2015 at the experimental farm of Mansoura Horticultural Research Station, Dakahlia governorate, Egypt to study the effect of yeast (1500 and $3000 \mathrm{ppm}$ ) as well as salicylic acid (10 and $20 \mathrm{ppm}$ ) on vegetative growth, essential oil percentage and oil yield of six celery local breed lines plants. Results revealed that foliar application with yeast and salicylic acid increased significantly the vegetative growth characters i.e plant height, number of lateral branches, number of leaves as well as plant fresh and dry weights were responded positively in all celery lines to the foliar application treatments of yeast and salicylic acid. Regardless the foliar application treatments, lines number 5 and 6 recorded the highest values of vegetative growth characters, while lines 1 and 2 gave the lowest values. Foliar application of salicylic acid or yeast improved fruit yield, essential oil percentage and oil yield recording values higher compared with control plants. Lines number 5 and 6 had higher yields in comparison to other used lines. Generally, it could be concluded that, the highest fruit and essential oil yields were found by foliar spraying line 6 with the highest concentration of yeast (3000 ppm).
\end{abstract}

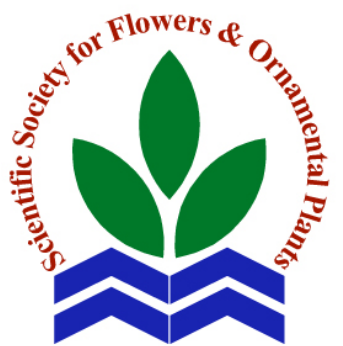

Scientific J. Flowers \& Ornamental Plants, 4(1):15-27(2017).

Received: 29/12/2016

Accepted: $16 / 1 / 2017$

Key words: Celery, yeast, salicylic acid, essential oil, fruit yield.

\section{INTRODUCTION}

Celery (Apium graveolens, L.) belonging to family Apiaceae (Umbelliferae) is an annual spicy herb and vegetable crop. The leaves are used fresh as salad or cooked with soup while, the fruits are used as spice and for medicinal purposes. It is known to increase the elimination of uric acid and is useful for gout, neuralgia and rheumatoid anthritis. It is antioxidative, antirheumatic antiseptic (urinary), antispasmodic, aperitif, depuratife, digestive, diuretic, carminative, nervine, sedative (nervous), stimulant (urine), stomachic, tonic (Lawless, 1992). Celery involved in the prevention of cardiovascular disease, lowering blood glucose in diabetic, lowering blood pressure and strengthening the heart (Momin and Nair, 2001 and Mencherini et al., 2007). Also, celery fruit is consumed as a diuretic, it is also used to treat symptoms of kidney, rheumatoid, and arthritis diseases. It is believed that celery seed tea gives relaxation and improves sleep. Celery fruits contain 2 to $3 \%$ essential oil. Its oil contains mostly limonene (usually 60 percent), selinene (10\%), frocoumarin and frocoumarin glycosides and their flavonoids.

Recently, great attention has been focused on the possibility of using natural and safety substituents for human and environment in order to improve the vegetative growth and yield of medicinal and aromatic plants. Salicylic acid and yeast are considered among these safe substances. 
Salicylic acid (SA) is an endogenous plant growth regulator, acting as non enzymatic antioxidant and has a regulatory effect on some physiological and biochemical processes, such as nutrients uptake, membrane stability, water relations, stomatal regulation, photosynthesis, growth and inhibition of ethylene biosynthesis (Khan et al., 2003 and Stevens et al., 2006) and induces flowering, increases flower life, retards senescence and increases cell metabolic rate (Metwally et al., 2003). Moreover, increased essential oil percentage and seed yield on coriander (Hesami et al., 2012 and 2013 and Said-Al Ahl et al., 2014).

Yeasts have been reported to be rich source of phytohormones (especially cytokinins), vitamins, enzymes, amino acids and minerals (Khedr and Farid, 2000). It was reported about its stimulatory effects on cell division and enlargement, protein and nucleic acid synthesis and chlorophyll formation (Castelfranco and Beale, 1983). Improving growth and productivity of medicinal and aromatic plants by application of active yeast extract were recorded by (ElSayed, 1985 on Achillia millifolium; Haridi, 1987 on Salvia officinalis; Refaat and Naguib, 1998 on peppermint; Ahmed, 1998; on marjoram; Naguib, 2002 on lemongrass; Heikal, 2005 on thyme; Seleim, 2005 on Mentha viridis and Salvia officinalis; Abd El-Latif, 2006 on Salvia officinalis; Ismail et al., 2007 on black cumin; Mady, 2009 on Majorana hortensis and Salvia officinalis; Ezz EL-Din and Hendawy, 2010 on borage and Ahmed et al., 2014 on basil).

Therefore, the present investigation was designed to disclose the influence of different levels from active yeast extract and salicylic acid on the growth, essential oil percentage and its yield of six lines of celery plants.

\section{MATERIALS AND METHODS}

The present study was carried out at ElBaramoon Research Farm, Mansoura Horticulture Research Station, HRI, ARC., Egypt through the two successive growing winter seasons of 2013/2014 and 2014/2015 to study the effect of foliar sprays with active yeast extract (1500 and $3000 \mathrm{ppm}$ ) and salicylic acid (10 and $20 \mathrm{ppm}$ ) on the growth, essential oil percentage and its yield of six lines of celery plants (resulted from breeding program at Horticulture Research Institute). The fruits of celery used in this research were secured from Cucurbits and Cross-Pollinated Vegetable Crops Research Department, Horticulture Research Institute, Agricultural Research Center, Giza, Egypt. Experimental design was laid in a split plot arrangement in completely randomized block design with three replications, and celery lines (6 lines) considered the main plots, spray treatments were assigned as sub plots. The fruits were sown in nursery in the first week of October in the two seasons and seedlings were transplanted in the permanent field in the first of December in both seasons on ridges $75 \mathrm{~cm}$ width at $30 \mathrm{~cm}$ apart. Each experimental plot was $2.25 \times 5 \mathrm{~m}$ and contained 3 ridges. The required agricultural practices for growing celery were followed as recommended. Soil sample was taken from the soil surface $(0-30 \mathrm{~cm})$, air dried, sieved by $2 \mathrm{~mm}$ sieve and analyzed for physical and chemical properties of soil according to Jackson, (1967) and the analysis results are presented in Table (1).

Spraying was performed using plastic atomizer after adding Tween $20(0.5 \%)$ as surfactant and plants were sprayed 5 times with Salicylic acid at 10 and $20 \mathrm{ppm}$ and Yeast extract at 1500 and 3000 ppm, untreated plants were left as a control and sprayed with distilled water, the first spraying was applied after 45 days from transplanting, the second after 2 weeks from the first and the last three sprays were after three cuts. After 75 days from transplanting during each growing season 5 plants/ replicate, were randomly selected to determine plant height $(\mathrm{cm})$, branch number/plant, number of leaves per plant and fresh and dry weights (g/plant). In addition, three cuts were taken to determine the leaf yield per plant and per feddan. At harvest stage (when the secondary umbels 
Table 1. Physical and chemical properties of the experimental site during the two growing seasons.

\begin{tabular}{|c|c|c|c|c|c|}
\hline \multirow{2}{*}{$\begin{array}{c}\text { Some physical } \\
\text { properties }\end{array}$} & \multicolumn{2}{|c|}{ Values } & \multirow{2}{*}{$\begin{array}{l}\text { Some chemical } \\
\text { Properties }\end{array}$} & \multicolumn{2}{|c|}{ Values } \\
\hline & $1^{\text {st }}$ season & $2^{\text {nd }}$ season & & $1^{\text {st }}$ season & $2^{\text {nd }}$ season \\
\hline Sand (\%) & 28.1 & 27.9 & pH* value $(1: 25)$ & 8.0 & 7.9 \\
\hline Silt (\%) & 31.8 & 31.6 & EC dSm ${ }^{-1}$ & 0.9 & 0.9 \\
\hline \multirow[t]{3}{*}{ Clay (\%) } & 40.1 & 40.5 & Total N (\%) & 0.03 & 0.04 \\
\hline & & & Available N (ppm) & & \\
\hline & & & $\mathrm{NH}_{4}-\mathrm{N}$ & 23.37 & 23.00 \\
\hline \multirow[t]{2}{*}{ Texture class } & Clay-loam & Clay-loam & $\mathrm{NO}_{2}-\mathrm{N}$ & 0.162 & 0.126 \\
\hline & & & NO3-N & 13.21 & 13.12 \\
\hline $\mathrm{CaCO}_{3}(\%)$ & 3.2 & 3.0 & Available P (ppm) & 13.3 & 12.6 \\
\hline Organic matter (\%) & 1.8 & 1.6 & Available K (ppm) & 304 & 302 \\
\hline
\end{tabular}

color was changed to green yellow), fruit yield per plant and per feddan were recorded. The volatile oil percentage was determined in the air dried fruits using a modified Clevenger apparatus according to Guenther, (1961) and oil yield per plant and per feddan were calculated.

Statistical analysis: All the collected data were tabulated and statistically analyzed by Statistical Analysis of variance using MSTAT-C version 4 (1987) software and the treatment means were compared using the LSD test according to Gomez and Gomez, (1984).

\section{RESULTS AND DISCUSSION}

\section{1- Vegetative growth:}

Data presented in Table (2) show that there are significant differences between different celery lines in vegetative growth characters in terms of plant height, number of branches per plant, number of leaves per plant and plant fresh and dry weights. Line No. 6 exhibited the highest values of all studied characters followed by line No. 5, line No. 3, line No. 4, line No. 1 and finally line No. 2. It was obvious from data that celery lines could be classified into 3 groups according to their growing vigor, Lines No. 5 and 6 are vigorous lines, lines No. 3 and 4 are moderate in growth while lines No.1 and 2 are weak in their growth. The same Table clearly shows that different sprayed treatments i.e. yeast concentrations (1500 and $3000 \mathrm{ppm}$ ) and salicylic acid at (10 and $20 \mathrm{ppm}$ ) in most cases significantly increased the estimated vegetative growth characteristics of celery in comparison to control. The best growth was obtained as a result of using yeast at $3000 \mathrm{ppm}$ and salicylic acid at $10 \mathrm{ppm}$. Concerning the interaction between lines and application treatments, it is clear that the best interaction in both seasons, was achieved when plants of line 6 were sprayed with yeast at $3000 \mathrm{ppm}$, followed by salicylic acid at $10 \mathrm{ppm}$ with the same line. However, the lowest values were obtained with line 2 or line 1 with control treatment (Table 3).

With regard to the stimulatory effect of yeast extract on celery growth characters, it could be attributed to the effect of yeast extract upon the endogenous phytohormones specially the growth promoters i.e. auxins, gibberellins and cytokinins (Nagodawithana, 1991). Also, the increase in the release of carbon dioxide through fermentation process effectively stimulates photosynthesis and accelerates the biosynthesis of carbohydrates. The beneficial effect of yeast on plant growth was supported by the results of Muller and Leopold (1966). It increases synthesis of plant growth promoters especially $\mathrm{GA}_{3}$, IAA and cytokinins which lead to improving cell division and cell enlargement (Moor, 1979). Improving growth characters of plant crops by application of active yeast extract were 
Table 2. Vegetative growth characters of different celery lines in response to yeast and salicylic acid foliar application during 2013/2014 and 2014/2015 seasons.

\begin{tabular}{|c|c|c|c|c|c|c|c|c|c|c|c|}
\hline \multirow{3}{*}{ Lines } & \multirow{3}{*}{$\begin{array}{l}\text { Treatments } \\
\text { Foliar } \\
\text { application }\end{array}$} & \multicolumn{2}{|c|}{$\begin{array}{l}\text { Plant height } \\
\text { (cm) }\end{array}$} & \multicolumn{2}{|c|}{$\begin{array}{c}\text { Number of } \\
\text { branches/plant }\end{array}$} & \multicolumn{2}{|c|}{$\begin{array}{l}\text { Number of } \\
\text { leaves/plant }\end{array}$} & \multicolumn{2}{|c|}{$\begin{array}{l}\text { Plant f.w. } \\
\text { (g) }\end{array}$} & \multicolumn{2}{|c|}{$\begin{array}{l}\text { Plant d.w. } \\
\text { (g) }\end{array}$} \\
\hline & & $1^{\text {st }}$ & $2^{\text {nd }}$ & $\mathbf{1}^{\text {st }}$ & $2^{\text {nd }}$ & $1^{\text {st }}$ & $2^{\text {nd }}$ & $1^{\text {st }}$ & $2^{\text {nd }}$ & $1^{\text {st }}$ & $2^{\text {nd }}$ \\
\hline & & Season & Season & Season & Season & Season & Season & Season & Season & Season & Season \\
\hline \multicolumn{12}{|c|}{ A: Lines } \\
\hline Line 1 & & 30.5 & 32.3 & 14.3 & 15.2 & 19.9 & 21.9 & 21.4 & 23.1 & 2.58 & 2.78 \\
\hline Line 2 & & 27.8 & 29.1 & 14.1 & 15.5 & 19.1 & 20.3 & 18.7 & 20.4 & 2.25 & 2.47 \\
\hline Line 3 & & 32.7 & 34.1 & 19.5 & 22.1 & 22.0 & 23.9 & 26.3 & 29.1 & 3.16 & 3.53 \\
\hline Line 4 & & 32.5 & 34.6 & 16.3 & 17.2 & 21.2 & 22.8 & 24.7 & 26.3 & 2.97 & 3.17 \\
\hline Line 5 & & 33.8 & 35.8 & 19.0 & 20.9 & 24.8 & 26.1 & 29.2 & 32.0 & 3.49 & 3.85 \\
\hline Line 6 & & 37.0 & 39.8 & 20.5 & 22.9 & 26.9 & 28.4 & 30.3 & 32.9 & 3.65 & 3.99 \\
\hline LSD 5\% & & 1.65 & 1.60 & 0.85 & 1.00 & 0.58 & 0.47 & 0.92 & 1.10 & 0.11 & 0.08 \\
\hline \multicolumn{12}{|c|}{ B: Foliar application } \\
\hline & Control & 28.8 & 30.8 & 14.0 & 16.2 & 19.0 & 20.7 & 21.6 & 23.9 & 2.61 & 2.90 \\
\hline & SA 10 ppm & 33.9 & 35.7 & 18.4 & 19.9 & 23.5 & 24.7 & 26.5 & 28.5 & 3.23 & 3.50 \\
\hline & SA 20 ppm & 31.3 & 33.1 & 16.4 & 17.9 & 21.1 & 22.8 & 23.8 & 26.1 & 2.78 & 3.04 \\
\hline & Yeast $1500 \mathrm{ppm}$ & 32.8 & 34.5 & 17.4 & 18.8 & 22.7 & 24.3 & 25.3 & 27.5 & 3.05 & 3.35 \\
\hline & Yeast $3000 \mathrm{ppm}$ & 35.2 & 37.4 & 20.2 & 21.9 & 25.3 & 26.8 & 28.4 & 30.6 & 3.42 & 3.70 \\
\hline & LSD $5 \%$ & 0.97 & 090 & 0.70 & 0.89 & 0.68 & 0.81 & 0.97 & 0.95 & 0.14 & 0.09 \\
\hline
\end{tabular}

recorded by Ahmed et al. (2014) on basil, Ismail et al. (2007) on black cumin, Naguib, (2002) on lemongrass, Naguib \& Khalil (2002) on black cumin, Ahmed et al. (1998) on roselle and Ahmed (1998) on marjoram.

Regarding salicylic acid, the promotive effects of salicylic acid on vegetative growth could be attributed to its bioregulator effects on physiological and biochemical processes in plants such as ions uptake, cell elongation, cell division, cell differentiation, sink/source regulation, enzymatic activities, protein synthesis and photosynthetic activity as well as increasing the antioxidant capacity of plants (El-Tayeb, 2005) as well as enhancing rooting of plants (SandovalYapiz, 2004), which plays a key role in enhancing the growth and productivity of plants. Moreover, the increase in plant height was due to the increase in number of internodes, while the increase in the fresh and dry weights of celery might be attributed to an increase in number of branches and leaves. Our results are in harmony with those of Said-Al Ahl et al. (2014); Hesami et al. (2012 and 2013) on coriander ; Rahimi et al. (2013) on cumin; Idrees et al. (2010) on lemongrass and Gharib (2006) on sweet basil and marjoram.

\section{2- Leaf and fruit yield:}

Data in Table (4) show clearly that leaf yield per plant and per feddan from the three cuts as well as fruit yield per plant and feddan were significantly affected by the different celery lines. The highest increments were the lines No. 6 and 5 in leaf yield 274 and $283 \mathrm{~g} /$ plant and 265 and $271 \mathrm{~g} /$ plant as well as fruit yield 881 and $902 \mathrm{~kg} /$ fed and 817 and $885 \mathrm{~kg} /$ fed for the first and second seasons, respectively. While, Line No. 2 was the lowest line, it gave $233.5 \mathrm{~g} / \mathrm{plant}$ and 
Table 3. Effect of the interaction between celery lines and foliar application treatments on vegetative growth characters during 2013/2014 and 2014/2015 seasons.

\begin{tabular}{|c|c|c|c|c|c|c|c|c|c|c|c|}
\hline \multirow{3}{*}{ Lines } & \multirow{3}{*}{$\begin{array}{l}\text { Treatments } \\
\text { Foliar application }\end{array}$} & \multicolumn{2}{|c|}{$\begin{array}{c}\text { Plant height } \\
\text { (cm) }\end{array}$} & \multicolumn{2}{|c|}{$\begin{array}{c}\text { Number of } \\
\text { branches/plant }\end{array}$} & \multicolumn{2}{|c|}{$\begin{array}{l}\text { Number of } \\
\text { leaves/plant }\end{array}$} & \multicolumn{2}{|c|}{$\begin{array}{l}\text { Plant f.w. } \\
\text { (g) }\end{array}$} & \multicolumn{2}{|c|}{$\begin{array}{l}\text { Plant d.w. } \\
\text { (g) }\end{array}$} \\
\hline & & $\mathbf{1}^{\text {st }}$ & $2^{\text {nd }}$ & $\mathbf{1}^{\mathrm{st}}$ & $2^{\text {nd }}$ & $1^{\text {st }}$ & $2^{\text {nd }}$ & $1^{\text {st }}$ & $2^{\text {nd }}$ & $\mathbf{1}^{\text {st }}$ & $2^{\text {nd }}$ \\
\hline & & Season & Season & Season & eason & Season & ason & Season & Season & Seaso & \\
\hline \multirow{5}{*}{ Line 1} & Control & 26.6 & 27.6 & 12.7 & 13.3 & 17.0 & 19.7 & 17.8 & 20.2 & 2.16 & 2.45 \\
\hline & SA 10 ppm & 32.0 & 33.4 & 14.3 & 16.3 & 21.0 & 22.7 & 22.6 & 23.8 & 2.76 & 2.90 \\
\hline & SA 20 ppm & 29.3 & 31.7 & 13.3 & 13.6 & 18.7 & 20.0 & 20.7 & 22.2 & 2.41 & 2.59 \\
\hline & Yeast $1500 \mathrm{ppm}$ & 30.0 & 32.0 & 13.6 & 14.0 & 19.7 & 21.6 & 21.3 & 22.6 & 2.57 & 2.73 \\
\hline & Yeast $3000 \mathrm{ppm}$ & 34.7 & 36.6 & 17.6 & 18.6 & 23.0 & 25.3 & 24.7 & 26.8 & 2.98 & 3.24 \\
\hline \multirow{5}{*}{ Line 2} & Control & 23.0 & 24.8 & 11.0 & 12.7 & 15.7 & 17.0 & 15.7 & 17.6 & 1.90 & 2.14 \\
\hline & SA 10 ppm & 29.3 & 29.7 & 15.3 & 16.0 & 20.7 & 21.7 & 20.7 & 22.2 & 2.52 & 2.71 \\
\hline & SA 20 ppm & 28.0 & 29.0 & 13.3 & 15.0 & 17.3 & 18.0 & 17.0 & 18.8 & 1.99 & 2.19 \\
\hline & Yeast $1500 \mathrm{ppm}$ & 29.0 & 29.5 & 14.7 & 16.3 & 20.0 & 21.0 & 18.8 & 20.2 & 2.28 & 2.44 \\
\hline & Yeast $3000 \mathrm{ppm}$ & 30.3 & 32.6 & 16.0 & 17.3 & 22.0 & 24.0 & 21.2 & 23.8 & 2.57 & 2.87 \\
\hline \multirow{5}{*}{ Line 3} & Control & 29.3 & 31.0 & 14.0 & 17.0 & 18.3 & 20.0 & 23.0 & 25.8 & 2.79 & 3.13 \\
\hline & SA 10 ppm & 34.0 & 35.0 & 21.3 & 23.3 & 23.0 & 24.0 & 27.4 & 30.1 & 3.34 & 3.67 \\
\hline & SA 20 ppm & 32.0 & 33.6 & 20.0 & 22.7 & 21.0 & 23.7 & 25.1 & 28.2 & 2.93 & 3.29 \\
\hline & Yeast $1500 \mathrm{ppm}$ & 33.3 & 35.0 & 20.3 & 22.7 & 22.7 & 25.0 & 26.3 & 29.9 & 3.17 & 3.61 \\
\hline & Yeast $3000 \mathrm{ppm}$ & 35.0 & 36.1 & 22.0 & 24.6 & 25.0 & 26.7 & 29.5 & 31.5 & 3.56 & 3.81 \\
\hline \multirow{5}{*}{ Line 4} & Control & 28.3 & 31.9 & 13.3 & 14.3 & 17.7 & 19.0 & 20.3 & 22.4 & 2.46 & 2.72 \\
\hline & SA 10 ppm & 34.1 & 37.0 & 18.0 & 19.0 & 22.3 & 23.7 & 26.1 & 27.4 & 3.19 & 3.34 \\
\hline & SA 20 ppm & 31.6 & 33.0 & 15.3 & 16.0 & 20.0 & 22.0 & 23.4 & 25.3 & 2.73 & 2.95 \\
\hline & Yeast $1500 \mathrm{ppm}$ & 33.0 & 34.3 & 16.0 & 16.6 & 21.6 & 24.0 & 25.8 & 27.0 & 3.12 & 3.26 \\
\hline & Yeast $3000 \mathrm{ppm}$ & 35.0 & 37.0 & 19.0 & 20.0 & 24.0 & 25.3 & 27.8 & 29.6 & 3.37 & 3.58 \\
\hline \multirow{5}{*}{ Line 5} & Control & 31.7 & 32.7 & 16.0 & 18.0 & 21.0 & 22.7 & 25.2 & 27.1 & 3.05 & 3.29 \\
\hline & SA 10 ppm & 35.4 & 37.7 & 19.3 & 21.7 & 26.0 & 27.0 & 30.5 & 33.6 & 3.71 & 4.10 \\
\hline & SA 20 ppm & 32.0 & 33.7 & 17.0 & 19.0 & 24.0 & 25.7 & 27.3 & 30.6 & 3.18 & 3.57 \\
\hline & Yeast $1500 \mathrm{p}$ & 33.5 & 36.0 & 20.3 & 21.0 & 25.3 & 26.3 & 29.2 & 32.8 & 3.53 & 3.96 \\
\hline & Yeast $3000 \mathrm{ppm}$ & 36.6 & 39.0 & 22.3 & 24 & 27.7 & 28.7 & 32.8 & 35.9 & 3.97 & 4.34 \\
\hline \multirow{5}{*}{ Line 6} & Control & 34.1 & 37.0 & 18.3 & 21. & 24.0 & 26.0 & 27.3 & 30.2 & 3.32 & 3.66 \\
\hline & SA 10 ppm & 38.7 & 41.6 & 22.0 & 23.3 & 28.0 & 29.3 & 31.4 & 33.8 & 3.83 & 4.13 \\
\hline & SA 20 ppm & 35.0 & 37.3 & 19.3 & 21.3 & 25.7 & 27.6 & 29.3 & 31.4 & 3.41 & 3.67 \\
\hline & Yeast $1500 \mathrm{ppm}$ & 37.7 & 40.0 & 19.6 & 22.0 & 27.0 & 28.0 & 30.1 & 33.0 & 3.64 & 3.99 \\
\hline & Yeast $3000 \mathrm{ppm}$ & 39.3 & 43.0 & 24.6 & 26.3 & 29.7 & 31.0 & 33.6 & 36.2 & 4.06 & 4.38 \\
\hline \multicolumn{2}{|c|}{ LSD $5 \%$} & 2.39 & 2.20 & 1.71 & 2.19 & 1.66 & 1.99 & 2.37 & 2.33 & 0.35 & 0.22 \\
\hline
\end{tabular}


Table 4. Effect of yeast and salicylic acid foliar application treatments on leaf and fruit yield of celery lines during 2013/2014 and 2014/2015 seasons.

\begin{tabular}{|c|c|c|c|c|c|c|c|c|c|}
\hline \multirow[b]{2}{*}{ Lines } & \multirow{2}{*}{$\begin{array}{l}\text { Treatments } \\
\text { Foliar } \\
\text { application }\end{array}$} & \multicolumn{2}{|c|}{$\begin{array}{l}\text { Leaf yield/plant } \\
\text { (g) }\end{array}$} & \multicolumn{2}{|c|}{$\begin{array}{c}\text { Leaf yield/fed } \\
\text { (ton) }\end{array}$} & \multicolumn{2}{|c|}{$\begin{array}{l}\text { Fruit yield/plant } \\
\text { (g) }\end{array}$} & \multicolumn{2}{|c|}{$\begin{array}{c}\text { Fruit yield/fed } \\
\text { (kg) }\end{array}$} \\
\hline & & $\begin{array}{c}1^{\text {st }} \\
\text { Season } \\
\end{array}$ & $\begin{array}{c}2^{\text {nd }} \\
\text { Season } \\
\end{array}$ & $\begin{array}{c}1^{\text {st }} \\
\text { Season } \\
\end{array}$ & $\begin{array}{c}2^{\text {nd }} \\
\text { Season } \\
\end{array}$ & $\begin{array}{c}1^{\text {st }} \\
\text { Season } \\
\end{array}$ & $\begin{array}{c}2^{\text {nd }} \\
\text { Season }\end{array}$ & $\begin{array}{c}1^{\text {st }} \\
\text { Season } \\
\end{array}$ & $\begin{array}{c}2^{\text {nd }} \\
\text { Season }\end{array}$ \\
\hline \multicolumn{10}{|c|}{ A: Lines } \\
\hline Line 1 & & 241 & 248 & 4.264 & 4.392 & 35.6 & 38.3 & 630 & 677 \\
\hline Line 2 & & 233 & 234 & 4.123 & 4.147 & 33.0 & 34.8 & 584 & 615 \\
\hline Line 3 & & 253 & 260 & 4.480 & 4.595 & 41.3 & 43.3 & 732 & 767 \\
\hline Line 4 & & 248 & 254 & 4.394 & 4.490 & 39.6 & 41.1 & 700 & 728 \\
\hline Line 5 & & 265 & 271 & 4.682 & 4.802 & 46.2 & 50.0 & 817 & 885 \\
\hline Line 6 & & 274 & 283 & 4.853 & 5.012 & 49.8 & 51.0 & 881 & 902 \\
\hline LSD 5\% & & 9.9 & 5.0 & 0.176 & 0.090 & 1.48 & 0.85 & 26.1 & 15.0 \\
\hline \multicolumn{10}{|c|}{ B: Foliar application } \\
\hline & Control & 225 & 233 & 3.986 & 4.128 & 30.3 & 32.5 & 535 & 576 \\
\hline & SA 10 ppm & 269 & 271 & 4.768 & 4.791 & 44.3 & 46.6 & 784 & 825 \\
\hline & SA 20 ppm & 237 & 243 & 4.196 & 4.298 & 34.8 & 37.4 & 616 & 662 \\
\hline & Yeast $1500 \mathrm{ppm}$ & 256 & 263 & 4.532 & 4.652 & 40.0 & 42.3 & 708 & 748 \\
\hline & Yeast $3000 \mathrm{ppm}$ & 274 & 282 & 4.847 & 4.997 & 55.2 & 56.5 & 977 & 1000 \\
\hline & LSD $5 \%$ & 5.8 & 4.1 & 0.104 & 0.074 & 1.00 & 0.71 & 17.7 & 12.6 \\
\hline
\end{tabular}

4.135 ton/fed for leaf yield as well as 33.9 g/plant and $599.5 \mathrm{~kg} / \mathrm{fed}$ for fruit yield (average both seasons). Moreover, yeast at $3000 \mathrm{ppm}$ was the superior treatment and gave 247 and $282 \mathrm{~g} /$ plant for leaf yield per plant and 4.853 and 5.012 ton/fed for leaf yield per feddan, 55.2 and $56.5 \mathrm{~g} /$ plant for fruit yield per plant and 977 and $1000 \mathrm{~kg} /$ fed for fruit yield per feddan for the first and second seasons, respectively. Salicylic acid at $10 \mathrm{ppm}$ came in the second rank without significant difference between it and yeast at $3000 \mathrm{ppm}$. On the contrary, control plants exhibited the lowest values of all leaf and fruit yield.

The interaction between celery lines and foliar application treatments showed significant differences in leaf and fruit yield per plant and per feddan as presented in Table (5). The high values of yield and its attributes were recorded when applying yeast at $3000 \mathrm{ppm}$ or salicylic acid at $10 \mathrm{ppm}$ to lines No. 6 or 5 plants. Spraying celery plants of line No. 6 with yeast at 3000 ppm increased the leaf yield per plant by $46 \%$ over control plants in line 2. Also increased the fruit yield per feddan by $146 \%$ as an average of the two seasons.

The positive effects of active dry yeast to plants attributed to their own contents of different nutrients, high protein and larger amount of vitamin B and natural plant growth regulators such as cytokinins which play a role in orientation and translocation of metabolites from leaves to the reproductive organs (Idso et al., 1995). Moreover, it might play a role in the synthesis of protein degradation which might lead to the improvement of yield and its quality (ElGhamriny et al., 1999). Improving growth and productivity of horticultural plants by application of active yeast extract were 
Table 5. Effect of the interaction between celery lines and foliar application of yeast as well as salicylic acid on leaf and fruit yield during 2013/2014 and 2014/2015 seasons.

\begin{tabular}{|c|c|c|c|c|c|c|c|c|c|}
\hline \multirow[b]{2}{*}{ Lines } & \multirow{2}{*}{$\begin{array}{l}\text { Treatments } \\
\text { Foliar application }\end{array}$} & \multicolumn{2}{|c|}{$\begin{array}{l}\text { Leaf yield/plant } \\
\text { (g) }\end{array}$} & \multicolumn{2}{|c|}{$\begin{array}{l}\text { Leaf yield/fed } \\
\text { (ton) }\end{array}$} & \multicolumn{2}{|c|}{$\begin{array}{c}\text { Fruit yield/plant } \\
\text { (g) }\end{array}$} & \multicolumn{2}{|c|}{$\begin{array}{c}\text { Fruit yield/fed } \\
\text { (kg) }\end{array}$} \\
\hline & & $\begin{array}{c}1^{\text {st }} \\
\text { Season } \\
\end{array}$ & $\begin{array}{c}2^{\text {nd }} \\
\text { Season }\end{array}$ & $\begin{array}{c}1^{\text {st }} \\
\text { Season } \\
\end{array}$ & $\begin{array}{c}2^{\text {nd }} \\
\text { Season }\end{array}$ & $\begin{array}{c}1^{\text {st }} \\
\text { Season } \\
\end{array}$ & $\begin{array}{c}2^{\text {nd }} \\
\text { Season }\end{array}$ & $\begin{array}{c}1^{\text {st }} \\
\text { Season } \\
\end{array}$ & $\begin{array}{c}2^{\text {nd }} \\
\text { Season }\end{array}$ \\
\hline \multirow{5}{*}{ Line 1} & Control & 210 & 217 & 3.721 & 3.843 & 25.4 & 28.8 & 450 & 510 \\
\hline & SA 10 ppm & 258 & 265 & 4.568 & 4.694 & 38.4 & 41.0 & 680 & 725 \\
\hline & SA 20 ppm & 220 & 229 & 3.894 & 4.053 & 29.3 & 32.0 & 519 & 567 \\
\hline & Yeast $1500 \mathrm{ppm}$ & 253 & 259 & 4.469 & 4.579 & 33.5 & 34.8 & 593 & 616 \\
\hline & Yeast $3000 \mathrm{ppm}$ & 264 & 271 & 4.669 & 4.793 & 51.1 & 54.7 & 904 & 969 \\
\hline \multirow{5}{*}{ Line 2} & Control & 204 & 208 & 3.611 & 3.676 & 23.8 & 25.9 & 422 & 458 \\
\hline & SA 10 ppm & 244 & 253 & 4.313 & 4.476 & 33.5 & 36.2 & 593 & 640 \\
\hline & SA 20 ppm & 211 & 216 & 3.726 & 3.823 & 26.8 & 28.1 & 474 & 497 \\
\hline & Yeast $1500 \mathrm{ppm}$ & 226 & 234 & 3.993 & 4.133 & 30.8 & 33.3 & 545 & 590 \\
\hline & Yeast $3000 \mathrm{ppm}$ & 256 & 261 & 4.528 & 4.625 & 50.0 & 50.4 & 884 & 891 \\
\hline \multirow{5}{*}{ Line 3} & Control & 225 & 233 & 3.983 & 4.124 & 29.3 & 32.4 & 519 & 574 \\
\hline & SA 10 ppm & 268 & 271 & 4.744 & 4.797 & 44.5 & 44.8 & 787 & 792 \\
\hline & SA 20 ppm & 241 & 246 & 4.257 & 4.352 & 36.5 & 38.2 & 646 & 676 \\
\hline & Yeast $1500 \mathrm{ppm}$ & 260 & 267 & 4.602 & 4.729 & 40.2 & 42.7 & 712 & 756 \\
\hline & Yeast $3000 \mathrm{ppm}$ & 272 & 281 & 4.814 & 4.974 & 57.5 & 58.5 & 1018 & 1035 \\
\hline \multirow{5}{*}{ Line 4} & Control & 215 & 220 & 3.813 & 3.894 & 28.9 & 31.3 & 511 & 554 \\
\hline & SA 10 ppm & 263 & 267 & 4.653 & 4.719 & 42.7 & 43.9 & 756 & 777 \\
\hline & SA 20 ppm & 235 & 239 & 4.156 & 4.237 & 34.0 & 36.1 & 602 & 640 \\
\hline & Yeast $1500 \mathrm{ppm}$ & 257 & 263 & 4.554 & 4.652 & 39.7 & 40.2 & 703 & 712 \\
\hline & Yeast 3000 ppm & 271 & 280 & 4.795 & 4.951 & 53.0 & 54.0 & 938 & 956 \\
\hline \multirow{5}{*}{ Line 5} & Control & 240 & 251 & 4.248 & 4.443 & 35.9 & 37.6 & 636 & 665 \\
\hline & SA 10 ppm & 275 & 277 & 4.868 & 4.903 & 50.6 & 56.9 & 896 & 1007 \\
\hline & SA 20 ppm & 254 & 259 & 4.490 & 4.575 & 39.0 & 45.0 & 690 & 796 \\
\hline & Yeast $1500 \mathrm{ppm}$ & 267 & 275 & 4.726 & 4.866 & 46.2 & 50.4 & 817 & 892 \\
\hline & Yeast $3000 \mathrm{ppm}$ & 287 & 295 & 5.076 & 5.222 & 59.4 & 60.1 & 1051 & 1064 \\
\hline \multirow{5}{*}{ Line 6} & Control & 257 & 271 & 4.542 & 4.788 & 38.1 & 39.2 & 675 & 693 \\
\hline & SA 10 ppm & 284 & 291 & 5.020 & 5.156 & 56.2 & 57.1 & 994 & 1011 \\
\hline & SA 20 ppm & 263 & 268 & 4.653 & 4.744 & 43.2 & 45.2 & 764 & 799 \\
\hline & Yeast $1500 \mathrm{ppm}$ & 274 & 280 & 4.850 & 4.956 & 50.6 & 52.1 & 895 & 922 \\
\hline & Yeast $3000 \mathrm{ppm}$ & 294 & 306 & 5.200 & 5.416 & 60.8 & 61.4 & 1077 & 1086 \\
\hline LSD 5\% & & 14.3 & 10.2 & 0.255 & 0.181 & 2.4 & 1.75 & 43.4 & 39.9 \\
\hline
\end{tabular}


recorded by Ahmed et al. (2014) on basil, Ismail et al. (2007) on black cumin, Naguib (2002) on lemongrass, Ahmed (1998) on marjoram and Ahmed et al. (1998) on rosella. Moreover, salicylic acid has roles in improving vegetative growth which affect positively on increasing the seed yield. There are promising results obtained when plants of Carica papaya were treated with salicylic acid which showed significantly higher fruit setting and subsequently increased the yield (Herrera-Tuz, 2004 and Martin-Mex et al., 2005). The present results are generally in agreement with those recorded by Said-Al Ahl et al. (2014); Hesami et al. (2012 and 2013) on coriander and Rahimi et al. (2013) on cumin.

\section{3- Essential oil percentage and yield:}

It is clear from Tables (6 and 7) that all tested concentrations of different foliar application treatments and the effect of celery lines as well as their interaction had significant effects on the percentage of essential oil and oil yield per plant (ml/plant) and per feddan ( $(/ /$ fed). It is clear that line No. 5, followed by line No. 6 and line 3 had the highest significant differences of essential oil percentages compared with other lines. On the contrary, line No. 2 had the lowest essential oil percentage. The same trend of essential oil percentage between lines was attained for essential oil yield per plant (ml/ plant) and per feddan (l/fed) as essential oil yield function to fruit yield and essential oil percentages.

Thus, as fruit yield and essential oil percentage increased subsequently essential oil yield was increased.

Table 6. Effect of different celery lines and foliar application treatments on celery essential oil percentage and oil yield during 2013/2014 and 2014/2015 seasons.

\begin{tabular}{|c|c|c|c|c|c|c|c|}
\hline \multirow[b]{2}{*}{ Lines } & \multirow{2}{*}{$\begin{array}{l}\text { Treatments } \\
\text { Foliar } \\
\text { application }\end{array}$} & \multicolumn{2}{|c|}{$\begin{array}{c}\text { Essential oil } \\
(\%)\end{array}$} & \multicolumn{2}{|c|}{$\begin{array}{c}\text { Essential oil } \\
\text { yield/ plant (ml) }\end{array}$} & \multicolumn{2}{|c|}{$\begin{array}{l}\text { Essential oil } \\
\text { yield/fed (l) }\end{array}$} \\
\hline & & $\begin{array}{c}1^{\text {st }} \\
\text { Season }\end{array}$ & $\begin{array}{c}2^{\text {nd }} \\
\text { Season }\end{array}$ & $\begin{array}{c}1^{\text {st }} \\
\text { Season }\end{array}$ & $\begin{array}{c}2^{\text {nd }} \\
\text { Season }\end{array}$ & $\begin{array}{c}1^{\text {st }} \\
\text { Season }\end{array}$ & $\begin{array}{c}2^{\text {nd }} \\
\text { Season }\end{array}$ \\
\hline \multicolumn{8}{|c|}{ A: Lines } \\
\hline Line 1 & & 1.23 & 1.27 & 0.447 & 0.492 & 7.909 & 8.709 \\
\hline Line 2 & & 1.13 & 1.16 & 0.382 & 0.413 & 6.754 & 7.306 \\
\hline Line 3 & & 1.35 & 1.38 & 0.565 & 0.603 & 9.989 & 10.670 \\
\hline Line 4 & & 1.30 & 1.33 & 0.519 & 0.551 & 9.184 & 9.760 \\
\hline Line 5 & & 1.41 & 1.45 & 0.655 & 0.728 & 11.596 & 12.886 \\
\hline Line 6 & & 1.5 & 1.53 & 0.752 & 0.784 & 13.317 & 13.882 \\
\hline LSD 5\% & & 0.05 & 0.02 & 0.023 & 0.016 & 0.410 & 0.280 \\
\hline \multicolumn{8}{|c|}{ B: Foliar application } \\
\hline & Control & 1.20 & 1.25 & 0.369 & 0.410 & 6.531 & 7.253 \\
\hline & SA 10 ppm & 1.38 & 1.41 & 0.621 & 0.665 & 10.991 & 11.764 \\
\hline & SA 20 ppm & 1.28 & 1.31 & 0.452 & 0.496 & 8.007 & 8.785 \\
\hline & Yeast $1500 \mathrm{ppm}$ & 1.30 & 1.33 & 0.528 & 0.572 & 9.354 & 10.127 \\
\hline & Yeast $3000 \mathrm{ppm}$ & 1.44 & 1.47 & 0.796 & 0.833 & 14.083 & 14.749 \\
\hline & LSD 5\% & 0.03 & 0.01 & 0.011 & 0.011 & 0.337 & 0.199 \\
\hline
\end{tabular}


Table 7. Essential oil percentage and oil yield as affected by the interaction between celery lines and yeast and salicylic acid foliar application during 2013/2014 and 2014/2015 seasons.

\begin{tabular}{|c|c|c|c|c|c|c|c|}
\hline \multirow[b]{2}{*}{ Lines } & \multirow{2}{*}{$\begin{array}{l}\text { reatments } \\
\text { Foliar application }\end{array}$} & \multicolumn{2}{|c|}{$\begin{array}{l}\text { Essential oil } \\
(\%)\end{array}$} & \multicolumn{2}{|c|}{$\begin{array}{c}\text { Essential oil } \\
\text { yield/plant (ml) }\end{array}$} & \multicolumn{2}{|c|}{$\begin{array}{l}\text { Essential oil } \\
\text { yield/fed (l) }\end{array}$} \\
\hline & & $\begin{array}{c}1^{\text {st }} \\
\text { Season }\end{array}$ & $\begin{array}{c}2^{\text {nd }} \\
\text { Season }\end{array}$ & $\begin{array}{c}1^{\text {st }} \\
\text { Season }\end{array}$ & $\begin{array}{c}2^{\text {nd }} \\
\text { Season }\end{array}$ & $\begin{array}{c}1^{\text {st }} \\
\text { Season }\end{array}$ & $\begin{array}{c}2^{\text {nd }} \\
\text { Season }\end{array}$ \\
\hline \multirow{5}{*}{ Line 1} & Control & 1.10 & 1.17 & 0.280 & 0.337 & 4.953 & 5.964 \\
\hline & SA 10 ppm & 1.29 & 1.30 & 0.496 & 0.533 & 8.775 & 9.427 \\
\hline & SA 20 ppm & 1.20 & 1.23 & 0.352 & 0.394 & 6.228 & 6.973 \\
\hline & Yeast 1500 ppm & 1.22 & 1.25 & 0.409 & 0.435 & 7.232 & 7.706 \\
\hline & Yeast 3000 ppm & 1.36 & 1.39 & 0.695 & 0.761 & 12.294 & 13.465 \\
\hline \multirow{5}{*}{ Line 2} & Control & 1.01 & 1.05 & 0.241 & 0.272 & 4.260 & 4.808 \\
\hline & SA 10 ppm & 1.21 & 1.26 & 0.405 & 0.456 & 7.177 & 8.069 \\
\hline & SA 20 ppm & 1.06 & 1.09 & 0.284 & 0.306 & 5.021 & 5.416 \\
\hline & Yeast $1500 \mathrm{ppm}$ & 1.08 & 1.10 & 0.333 & 0.367 & 5.886 & 6.489 \\
\hline & Yeast 3000 ppm & 1.30 & 1.32 & 0.649 & 0.665 & 11.496 & 11.766 \\
\hline \multirow{5}{*}{ Line 3} & Control & 1.23 & 1.27 & 0.361 & 0.412 & 6.381 & 7.290 \\
\hline & SA 10 ppm & 1.43 & 1.44 & 0.636 & 0.645 & 11.253 & 11.411 \\
\hline & SA 20 ppm & 1.32 & 1.34 & 0.481 & 0.512 & 8.521 & 9.060 \\
\hline & Yeast 1500 ppm & 1.33 & 1.36 & 0.535 & 0.581 & 9.463 & 10.279 \\
\hline & Yeast 3000 ppm & 1.45 & 1.48 & 0.834 & 0.865 & 14.765 & 15.314 \\
\hline \multirow{5}{*}{ Line 4} & Control & 1.21 & 1.24 & 0.350 & 0.388 & 6.187 & 6.870 \\
\hline & SA 10 ppm & 1.37 & 1.39 & 0.585 & 0.610 & 10.352 & 10.801 \\
\hline & SA 20 ppm & 1.24 & 1.27 & 0.421 & 0.459 & 7.460 & 8.122 \\
\hline & Yeast $1500 \mathrm{ppm}$ & 1.27 & 1.30 & 0.504 & 0.523 & 8.924 & 9.250 \\
\hline & Yeast 3000 ppm & 1.40 & 1.44 & 0.742 & 0.778 & 13.133 & 13.764 \\
\hline \multirow{5}{*}{ Line 5} & Control & 1.30 & 1.35 & 0.467 & 0.507 & 8.263 & 8.973 \\
\hline & SA 10 ppm & 1.45 & 1.48 & 0.734 & 0.842 & 12.992 & 14.900 \\
\hline & SA 20 ppm & 1.39 & 1.41 & 0.542 & 0.634 & 9.595 & 11.221 \\
\hline & Yeast $1500 \mathrm{ppm}$ & 1.40 & 1.44 & 0.646 & 0.725 & 11.438 & 12.841 \\
\hline & Yeast 3000 ppm & 1.50 & 1.55 & 0.891 & 0.932 & 15.765 & 16.491 \\
\hline \multirow{5}{*}{ Line 6} & Control & 1.36 & 1.39 & 0.519 & 0.544 & 9.181 & 9.635 \\
\hline & SA $10 \mathrm{ppm}$ & 1.55 & 1.58 & 0.870 & 0.902 & 15.405 & 15.974 \\
\hline & SA 20 ppm & 1.47 & 1.49 & 0.635 & 0.673 & 11.238 & 11.907 \\
\hline & Yeast $1500 \mathrm{ppm}$ & 1.51 & 1.54 & 0.764 & 0.802 & 13.519 & 14.196 \\
\hline & Yeast 3000 ppm & 1.60 & 1.63 & 0.973 & 1.000 & 17.227 & 17.703 \\
\hline LSD 5\% & & 0.08 & 0.08 & 0.047 & 0.042 & 0.830 & 0.690 \\
\hline
\end{tabular}


It could be pointed out that exogenous application of yeast and salicylic acid had a marked role and positive effects on the essential oil percentage and yield in celery plants. Yeast at $3000 \mathrm{ppm}$ was the best treatment for increasing essential oil percentage and its yield followed by salicylic acid at $10 \mathrm{ppm}$, yeast at $1500 \mathrm{ppm}$ and salicylic acid at $20 \mathrm{ppm}$. In addition, application of yeast at $3000 \mathrm{ppm}$ doubled the essential oil yield per plant when compared with control plants $(0.796$ and $0.833 \mathrm{ml} /$ plant versus 0.369 and $0.410 \mathrm{ml} /$ plant) for the first and second seasons, respectively.

As regard to the interaction between different celery lines and foliar application treatments and their effects on essential oil percentage and yield, data presented in Table (7) indicated that cultivation of lines No. 6 or 5 and spraying them with yeast at $3000 \mathrm{ppm}$ or salicylic acid at $10 \mathrm{ppm}$ increased essential oil percentage and yield. The highest values of essential oil percentage and yield were obtained from plants of the interaction between line No. 6 with yeast at 3000 ppm, followed by plants of line 5 with yeast at 3000 ppm, plants of line 6 with salicylic acid at $10 \mathrm{ppm}$ and then plants of line 5 with salicylic acid at $10 \mathrm{ppm}$. On the other hand, control plants of line 2 gave the lowest values for essential oil percentage and oil yield per plant and per feddan.

Concerning the promotive effect of yeast on essential oil percentage and oil yield, Subba Rao (1984) and Dewic (2000) mentioned that these results may be due to the stimulatory effect of yeast which acts as co-enzymes in photosynthesis and metabolism of carbohydrates and other metabolites. Our results on yeast agreed with those obtained earlier by Ahmed et al. (2014) on basil, Heikal (2005) on thyme, Selim (2005) on Mentha viridis and Salvia officinalis, Naguib (2002) on lemongrass, Ahmed et al. (1998) on roselle, Ahmed (1998) on marjoram, Refaat and Naguib (1998) on peppermint and Haridi (1987) on Salvia officinalis. The positive effects of salicylic acid on essential oil yield and components may be due to its role in increasing cell metabolism, affecting plastid and pathways of secondary metabolites and enhancing the synthesis of phenolic compounds (Gorni and Pacheco 2016 and Talaat et al., 2013). Idrees et al. (2010) stated that the improvement in essential oil content by foliar application of SA might be due to the increase in cycle growth, nutrients uptake or changes in leaf oil gland population and monoterpenes biosynthesis. Improvement of essential oil percentage and yield per plant in response to SA application has been reported in other plant species (Gharib 2006; Idrees et al. 2010). The obtained results of salicylic acid were in the same line with those of Arzandi (2014) and Said-Al Ahl et al. (2014) on coriander and Rahimi et al. (2013) on Cuminum cyminum.

\section{CONCLUSION}

It was observed from this study that, using foliar application of yeast at $3000 \mathrm{ppm}$ and or salicylic acid at $10 \mathrm{ppm}$ stimulated most of vegetative growth characters led to producing higher total yield and essential oil content in celery plants.

\section{REFERENCES}

Abd El-Latif, E.S.M. (2006). Effect of Chemical, Organic Fertilizers and Spraying With Active Dry Yeast on Growth, Oil Production and Plant Constituents of Sage (Salvia officinalis, L.) Plant. M.Sc. Thesis, Fac. Agric., Cairo Univ., Egypt.

Ahmed, E.T. (1998). Influence of concentration and time of spraying active dry yeast on growth and volatile oil content of marjoram plant. J. Agric. Sci., Mansoura Univ., 23(11):5067-5081.

Ahmed, S.K.; El-Ghawas, E.O. and Aly, A.F. (1998). Effect of active dry yeast and organic manure on roselle plant. Egyptian J. Agric. Res., 76(3):11151142.

Ahmed, S.K.; Hammam, Kh. A. and Amer, A.A. (2014). Effect of bio-fertilization and some plant extracts on the growth, 
yield and chemical constituents of basil plant. J. Plant Production, Mansoura Univ., 5(2):193-210.

Arzandi, B. (2014). The effect of salicylic acid different levels on two Coriandrum sativum varieties under deficit irrigation condition. European Journal of Zoological Research, 3(1):118-122.

Castelfranco, P.A. and Beale, S.I. (1983). Chlorophyll biosynthesis: recent advances and area of current interest. Ann. Rev. Plant physiology, 34:241-278.

Dewic, K.P.M. (2000). Medicinal Natural Products, A Biosynthetic Approach. $2^{\text {nd }}$ Ed. John Wiley \& Sons, N.Y., pp. 306356.

El-Ghamriny, E.A.; Arisha, E.A.H. and Nour, K.A. (1999). Studies on tomato flowering, fruit set, yield and quality in summer season. I. Spraying with thiamine, ascorbic acid and yeast. Zagazig J. Agric. Res., 26(5):1345-1364.

El-Sayed, A.A. (1985). Effect of Nitrogen Fertilization and Some Growth Regulators on Yarrow (Achillea millefolium, L.) Plants. M.Sc. Thesis, Fac. Agri., Minia Univ., Egypt.

El-Tayeb, M.A. (2005). Response of barley grains to the interactive effects of salinity and salicylic acid. Plant Growth Regul., 45:215- 224.

Ezz EL-Din, A. and Hendawy, S.F. (2010). Effect of dry yeast and tea compost on growth and oil content of Borago officinalis plants. Research Journal of Agriculture and Biological Science, 6(4): 424-430.

Gharib, F.A.E. (2006). Effect of salicylic acid on the growth, metabolic activities and oil content of basil and marjoram. Int. J. Agric. Biol., 4:48-492.

Gomez, K.A. and Gomez, A.A. (1984). Statistical Proceedings for Agricultural Research. Second Edition. John Wiley, New York.
Gorni, P.H. and Pacheco, A.C. (2016). Growth promotion and elicitor activity of salicylic acid in Achillea millefolium L. African Journal of Biotechnology, 15(16):657-665.

Guenther, G. (1961). The Volatile Oils VIII. Robert E.D. Nastrand Comp. Inc. Toronto, New York, London.

Haridi, S.M.M. (1987). Biochemical Studies on Sage Salvia officinalis Plants. Ph.D. Thesis., Ain Shams Univ., Egypt.

Heikal, A.A.M. (2005). Effect of Organic and Bio-Fertilization on Growth, Production and Composition of Thymus vulgaris, L. Plants. M.Sc.Thesis, Faculty of agriculture, Cairo Univ., Egypt.

Herrera-Tuz, R. (2004). Reguladores de crecimiento XXI. Efecto del acido salicilico en la productividad de papaya maradol (Carica papaya L.). Tesis de Licenciatura. Instituto Tecnologico Agropecuario, Conkal, Yucatan, Mexico.

Hesami, S.; Nabizadeh, E.; Rahimi, A. and Rokhzadi, A. (2012). Effects of salicylic acid levels and irrigation intervals on growth and yield of coriander (Coriandrum sativum) in field conditions. Environmental and Experimental Biology, 10: 113-116.

Hesami, S.; Rokhzadi, A.; Rahimi A.; Hesami, G. and Kamangar, H. (2013). Coriander response to foliar application of salicylic acid and irrigation intervals. International Journal of Biosciences, 3(11):35-40.

Idrees, M.; Khan, M.M.A.; Aftab, T.; Naeem, M., and Hashmi, N., (2010). Salicylic acid-induced physiological and biochemical changes in lemongrass varieties under water stress. J. Plant Interact. 5: 293-303

Idso, S.B.; Idso, K,E. and Hoober, J.K. (1995). Effect of atmospheric $\mathrm{CO}_{2}$ enrichment and foliar methanol application on net photosynthesis of sour orange trees (Citrus aurantium) leaves. Amer. J. Botany, 82 (1):26-30. 
Ismail, S.I.; Khafagy, M.A. and Khater, M. R. (2007). Improvement of growth and yield of black cumin plant by active dry yeast and some vitamins. J. Agric. Sci., Mansoura Univ., 32(11):8977-8989.

Jackson, M.L. (1967). Soil Chemical Analysis. Printice-Hall of India Private Limited, New Delhi: 144-197.

Khan, W.; Prithiviraj, B., and Smith. D.L. (2003). Photosynthetic responses of corn and soybean to foliar application of salicylates. J. Plant Physiol., 160:485492.

Khedr, Z.M.A. and Farid, S. (2000). Response of naturally infected tomato to yeast extract and phosphoric acid application. Annals of Agric. Sci., Moshtohor, 38(2):927-393.

Lawless, J. (1992). Encyclopedia of Essential Oils. Published in "Element", Inc., 24 Broadway, Rockport, MAO1966., p. 11-15, 52-54 and 126128.

Mady, A. (2009). Effect of Certain Medicinal Plant Extracts on Growth, Yield and Metabolism of Some Medicinal and Aromatic Plants. M.Sc., Al-Azher Univ., Fac. Sci., Botany and Microbiology Dep., Egypt.

Martin-Mex, R.; Nexticapan-Garces, A., and Larque-Saavedra, A. (2005). Effect of salicylic acid in sex expression in Carica papaya L. In: $10^{\text {th }}$ International Symposium on Plant Biorregulators in Fruit Production, June 26-30 de 2005. Saltillo, Coahuila, Mexico, p. 113.

Mencherini, T.; Cau, A., Bianco, G.; Della Loggia, R.; Aquino, R.P. and Autore, G. (2007). An extract of Apium graveolens var. dulce leaves: structure of the major constituent, apiin, and its antiinflammatory properties. J. Pharm. Pharmacol., 59(6):891-7.

Metwally, A.; Finkemeier, I.; Georgi, M. and Dietz, K.J. (2003). Salicylic acid alleviates the cadmium toxicity in barley seedlings. Plant Physiol., 132: 272-81.
Momin, R.A. and Nair, M.G. (2001). Mosquitocidal, nematicidal, and antifungal compounds from Apium graveolens L. seeds. J. Agric. Food Chem., 49(1):142-5.

Moor, T.C. (1979). Biochemistry and Physiology of Plant Hormones. Pub. by Springer-Verlag, New York, USA.

MSTAT-C Version 4 (1987). Software Program for the Design and Analysis of Agronomic Research Experiments. Michigan University, East Lansing, Michigan, USA.

Muller, K. and Leopold. A.C. (1966). Correlative again and transport of pincom leaves under influence of kinetin. Planta, 68:167-185.

Nagodawithana, W.T. (1991). Yeast Technology. Universal Foods Corporation Milwaukee, Wisconsin Published by Van Nostrand Reinhold, New York.. 273 pp.

Naguib, N.Y. (2002). Yield and quality of lemongrass plants (Cymbopogon flexuous Stapf) as influenced by farm yard manure and foliar application of bread yeast. Annals of Agric. Sci., Cairo Univ., 47(3):859-873.

Naguib, N.Y. and Khalil, M.Y. (2002). Studies on the effect of dry yeast, thiamine and biotin on the growth and chemical constituents of black cumin (Nigella sativa L.). J. Agric. Sci., Arab Univ., 10(3):919-937.

Rahimi, A.R.; Rokhzadi, A.; Amini, S. and Karami, E. (2013). Effect of salicylic acid and methyl jasmonate on growth and secondary metabolites in Cuminum cyminum L. Journal of Biodiversity and Environmental Sciences., 3(12):140-149.

Refaat, A.M. and Naguib, N.Y. (1998). Peppermint yield and oil quality as affected by application of some amino acids. Bull. Fac. Agri., Cairo Univ., 49:89-98. 
Said Al-Ahl, H.A.H.; El Gendy, A.G. and Omer, E.A. (2014). Effect of ascorbic acid, salicylic acid on coriander productivity and essential oil cultivated in two different locations. Adv. Environ. Biol., 8(7):2236-2250.

Sandoval-Yapiz, M.R., (2004). Reguladores de crecimiento XXIII: efecto del acido sali- cilico en la biomasa del cempazuchitl (Tagetes erecta). Tesis de Licenciatura. Instituto Tecnologico Agropecuario, Conkal, Yucatan, Mexico.

Seleim, M.K.M. (2005). Physiological Studied on Some Medicinal Plants. M.Sc. Thesis, Fac. Agric. Tanta Univ., Egypt.

Stevens, J.; Senaratna, T. and Sivasithamparam, K., (2006). Salicylic acid induces salinity tolerance in tomato (Lycopersicon esculentum cv. Roma): associated changes in gas exchange, water relations and membrane stabilisation. Plant Growth Regul., 49: 77-83.

Subba Rao, N.S. (1984). Biofertilizers in Agriculture. Oxford. IBH Company, New Delhi., pp: 189.

Talaat, I. M.; Khattab, H.I. and Ahmed, A. M. (2013). Changes in growth, hormone levels and essential oil content of Ammi visnaga plants treated with some bioregulators. Nusantara Biosci Ence 5(2):57-64.

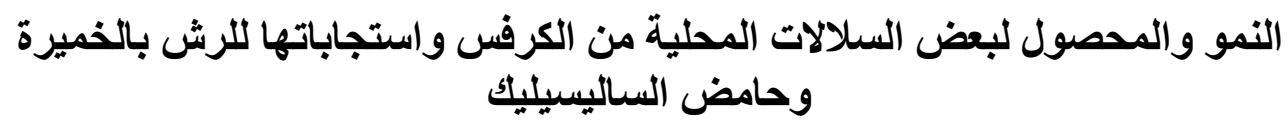

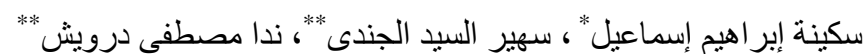

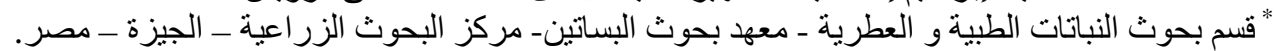

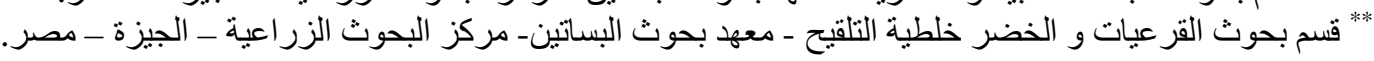

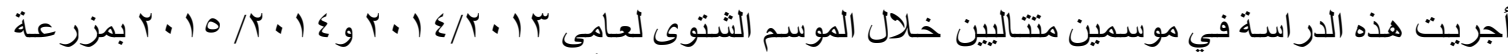

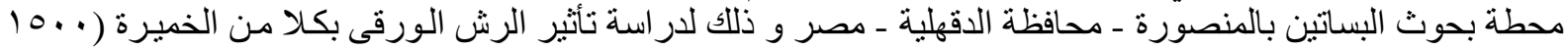

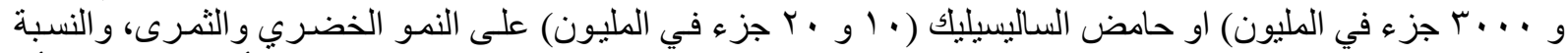

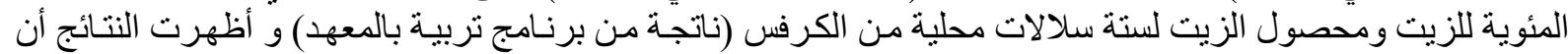

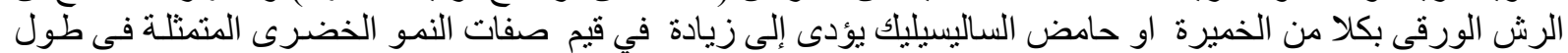

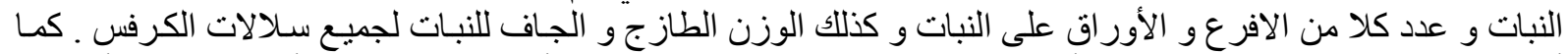

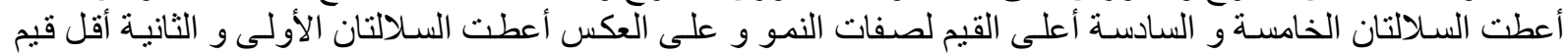

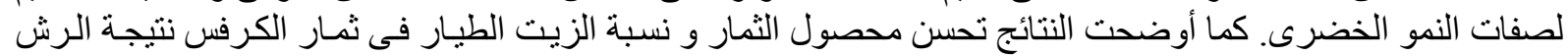

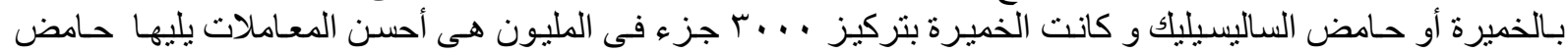

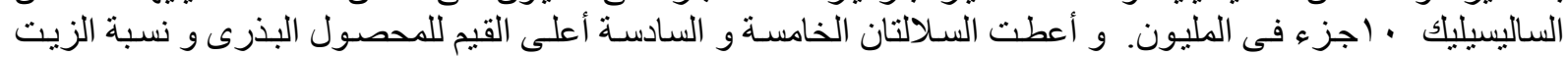

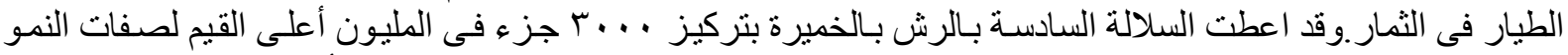

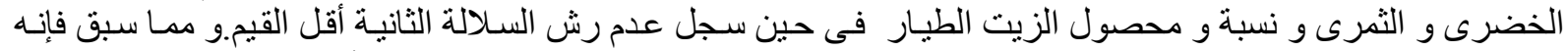

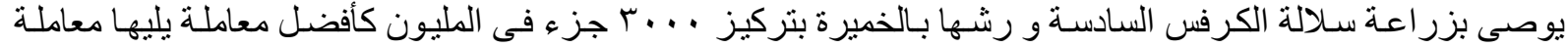
الرش بحامض الساليسيليك بتركيز • جز ء فى المليون و ذللك للحصول على أعلى محصول من الكرفس. 


